M. Al-Aboodi and I. A. Lami, "OCBPSR: Orthogonal Complex BandPass Sampling Receiver," presented at the 2014 World Congress on Computer Applications and Information Systems (WCCAIS), Hammamet, Tunisia, 2014.

(C) 2014 IEEE. Personal use of this material is permitted. Permission from IEEE must be obtained for all other uses, in any current or future media, including reprinting/republishing this material for advertising or promotional purposes, creating new collective works, for resale or redistribution to servers or lists, or reuse of any copyrighted component of this work in other works. 


\title{
OCBPSR: Orthogonal Complex BandPass Sampling Receiver
}

\author{
Maher AL-Aboodi and Ihsan Alshahib Lami \\ Applied Computing Department \\ The University of Buckingham, UK \\ First.last@buckingham.ac.uk
}

\begin{abstract}
Second-order/Complex BandPass Sampling Receivers (CBPSR) are attractive for acquiring multi-signals for SDR/CR applications. One of the issues caused by the implementation of such receivers is the signals IQ mismatch. This paper proposes a CBPSR implementation that eliminates this IQ mismatch by reformatting the received signals in an orthogonal analytic form (thus named OCBPSR). Our implementation will also reduce the required sampling frequency to below the Nyquist rate, and so reducing the processing time to recover the signals. This is achieved by folding the upper-side of the received signals to the same fold-frequency in the baseband domain without overlapping, by making the signals orthogonal. MATLAB simulation is used to evaluate the performance of our OCBPSR using various scenarios of harsh signal environment, including Doppler and multipath effects.
\end{abstract}

Keywords-component; Orthogonal signals, BPSR, multi-signal receiver Introduction, Fading channel, multipath, RLS and LMS.

\section{INTRODUCTION \& RELATED WORK}

Multi-signal receivers/transmitters are highly desirable in Smartphones because they come with many wireless technologies such GSM, LTE, Wi-Fi, Bluetooth and GNSS. Therefore shared components of the received/transmit signal chain functions will help saving silicon size \& cost as well as reducing processing time and thus battery power consumption. Multi-signal processing of GNSS signals, for example, will also help improving the overall tracking [1] and detection [2] capability when compared with single-signal GPS receiver.

The integration of wireless transceivers is a major research area due to the take off of software defined radio (SDR) and cognitive radio (CR) [3] solutions. i.e. The keystone of SDR and CR technology is how to build a flexible front-end architecture that can handle multiple signals simultaneously. Challenges of the conventional front-end architectures include the narrow-bandwidth of IF filters, frequency range of local oscillator and the unwanted signal images that come from the analogue mixers [4].

Bandpass sampling (BPS) provides a front-end technique where analogue multi-bandpass signals are downconverted (or folded) to baseband (or near baseband) without utilising an analogue mixer, local oscillator or image filters. This is achieved by bringing the Analogue-to-Digital Converter (ADC) as close as possible to the antenna. i.e. as well as handling multi-signals at the same time, the signals are sampled at a frequency which is proportional to the bandwidth information of the input signals rather than the highest frequency [5]. Therefore, the sampling frequency becomes a tiny proportion of the carrier frequency of the input signals; thus reducing the samples processing and in turn reducing the power consumption.

A first order implementation of a BPS (real-sampling) receiver requires a sampling frequency greater than double of the summation of bandwidths of the input signals. Also, the folded bandwidths of these input signals in first Nyquist zone (FNZ) must not overlap with themselves or with others [6], or folded to the reference frequency at $0 \mathrm{~Hz}$. Note that, it is also not possible to use aliasing to translate the bandwidth information of input signals directly to baseband. These limitations of the real-sampling receiver can be relaxed by using the idea of complex sampling, also called second-order BPS. Such implementation would use an "analytic signal" that is obtained by a $90^{\circ}$ phase shifting (using Hilbert transform (HT)), resulting in two sampled components; the I-component and the Q-component. An "analytic signal" means that only a single-side band of any double-band signals is actually processed by this second-order sampling receiver.

As shown in Fig.1, this is achieved by splitting the received signals into two paths. The Q-component path passes through an HT filter $\left(90^{\circ}\right.$ phase shifting) before an ADC, while the Icomponent path of the signal is passed to an ADC directly and then both paths are recombined. This will allow the ADC's to sample the signals with sampling frequency greater than the summation of the input signals' bandwidths. In this sampling, it is possible to fold the bandwidth information of the input signals directly at zero-folding-frequency (or baseband) [7]. However, implementations of such second-order BPS suffers from an I/Q imbalance, which results in imperfect image rejection (negative band). i.e., the resulting image signal of the negative band will also be located in the desired signal band, and therefore, directly degrades the bit error rate (BER) and the error vector magnitude (EVM).

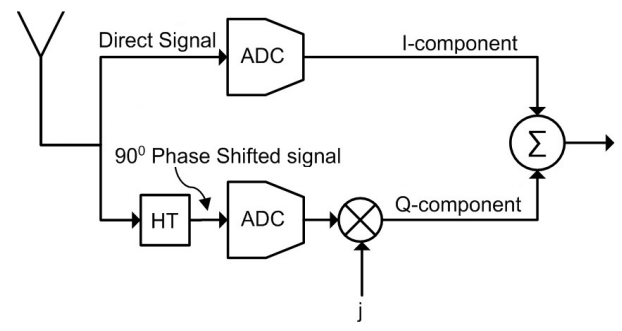

Figure 1. Complex BandPass Sampling Receiver (CBPSR) 
The Polyphase filtering technique can be used to reduce this IQ mismatch problem [8]. However, extra signal processing is required that takes away the ease-ofimplementation characteristics of typical front-end receivers. Sampling the Q-path of the signal at specific time-delay $\left(1 / 4 f_{c}\right)$ is another proposed implementation [12] for single signal direct conversion receivers. This sampling technique produces a fixed $90^{\circ}$ phase shift so to completely eliminate the mismatch between I and $\mathrm{Q}$ by eliminating the image of a specific carrier frequency (fc) that is used in the time-delay.

Several digital compensation methods are also proposed for solving IQ mismatch issues. Adaptive algorithms, such as maximum-likelihood [9] and least-squared estimation [10], are employed to estimate the IQ mismatch based on using training signals (these includes extra bits added to the transmitted signal that are solely used for synchronising the received signal with the generated signal). However, such training signals cost additional processing. To overcome this, Blind-compensation Algorithms are also used, such as the "source equivariant adaptive separation algorithm" [11] and the "de-convolution algorithm" [12]. These algorithms are typically implemented in both the I and the Q paths of the signal, and estimates the phase and the gain that have been induced by each path process and will be compensated accordingly.

Our OCBPSR implementation solves the IQ mismatch problem for two signals by harmonising any these received signals to an orthogonal form. As shown in Fig.2, this is achieved by feeding one of the two signals to an HT and then recombining the two signals after the ADCs. Our implementation produces the same time-delay (phase \& gain) to the I\&Q of the shifted signal. On the other hand, the first signal will not suffer from the time-delay because it is digitised directly. Furthermore, the OCBPSR also reduces the sampling rate to be proportional to the maximum bandwidth of the received signals instead of the summation of the two signal bandwidths as required by typical second-order BPS receivers. This will significantly reduce the processing time in the digital domain and therefore reducing the power consumption [13].

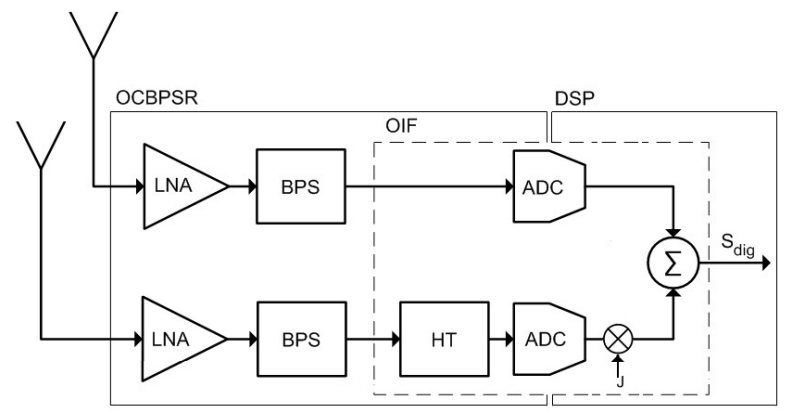

Figure 2. Orthogonal Complex BandPass Sampling Receiver (OCBPSR)

The rest of this paper is organised as follows: Section II contains the main concepts of the $1^{\text {st }}$ and $2^{\text {nd }}$ order BPS techniques. In section III, the description and mathematical representation of our proposed OCBPSR are detailed. While in section IV, the fading channel is explained as used in our receiver. Section $\mathrm{V}$ describes the simulation \& results of the experimental set-up that has been used to evaluate OCBPRS. Section VI concludes this work.

\section{BPS TECHNIQUE}

\section{A. Basics of BPS}

It is possible to use a signal sampling rate below the Nyquist frequency when using the BPS technique, without aliasing the information signals band. Here, it is a condition to choose a suitable sampling rate so to prevent overlapping of the folded signal with itself in any one of the Nyquist zones. Fig. 3 shows the frequency domain representation of BPS technique with non-overlapped folded sampled signals. For a single signal, the folding/aliasing-free process is a convolution operation between the FFT of the received signal and the summation of the shifted direct-delta function of its sampled pulses. This convolution results in a discrete time \& amplitude signal with a periodic-spectrum, where the "original continuous-time spectrum" is repeated at all integer-multiple of the sampling-frequency (or Nyquist Zones). i.e. any of these periodic-spectrum replicas/images in any of the NZs contains the full information of the original signal band, and thus can be used to recover the signal or for further processing.

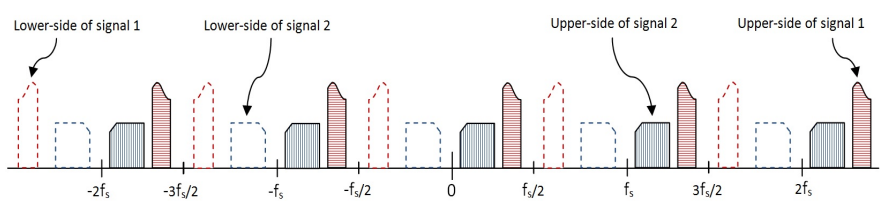

Figure 3. Frequncy domain representation of BPS for two signals

\section{B. $1^{\text {st }}$ order BPS (Real Sampling)}

Generally, the proper sampling frequencies are a function of both the bandwidth and the centre frequency of the desired signal. These proper sampling frequency intervals can be expressed as:

$$
\frac{2 \mathrm{f}_{\mathrm{c}}+\mathrm{B}}{\mathrm{k}+1} \leq \mathrm{f}_{\mathrm{s}} \leq \frac{2 \mathrm{f}_{\mathrm{c}}-\mathrm{B}}{\mathrm{k}}
$$

Where; $\mathrm{k}$ is an integer number bounded between 0 and normalize carrier frequency $\left(f_{c} / B-0.5\right), f_{s}$. is the sampling frequency, and $\mathrm{B}$ is the bandwidth of the signal.

In general, the bandwidth of the bandpass signal (real signal) is $(-0.5 \mathrm{~B} \leq \mathrm{f} \leq 0.5 \mathrm{~B})$; therefore the minimum sampling frequency for alias free in real sampling is $f_{s}=2 B$. Further details are available in [14].

\section{C. $2^{\text {nd }}$ order BPS (Complex Sampling)}

A sampled complex/analytic signal is one that has a nonzero spectrum only at frequencies $\left(\mathrm{f}_{\mathrm{c}}-\frac{\mathrm{B}}{2} \leq \mathrm{f} \leq \mathrm{f}_{\mathrm{c}}+\frac{\mathrm{B}}{2}\right)$ and sampling rate $\mathrm{f}_{\mathrm{s}}=\mathrm{B}$. Complex/analytic sampling will eliminate the negative spectrum (lobe) of the signals, thus the samples into half and the signal will not overlap with itself. Therefore, we can fold any signal directly to the zero fold-frequency (baseband).

\section{OCBPSR}

As shown in Fig. 2, our proposed 2-signals OCBPSR architecture consists of two Low-Noise Amplifiers (LNA), two BPFs, one $90^{\circ}$ phase-shifter, and two ADC's; each one of them is specified for the first and second received signals. The phase shifters (HT) and the ADC's make up the Orthogonal 
Integrated Function (OIF) [15] used for reforming the orthogonality of the filtered received signals. The HT is used as the first stage of OIF for shifting the phase of the second received signal by $90^{\circ}$ to prevent the signals overlapping prior to using the BPS technique. By choosing an appropriate sampling frequency, the second stage of OIF uses a BPS technique to fold both received signals directly to the same fold-frequency in the FNZ, thus producing a complex orthogonal signal (analytic signal) that comprises the two input signals. The mathematical representation of the signal is shown in (4). It is clear that the signals are orthogonalised at the folded frequency. After removing the carriers of the two signals and then recombining the I and the $\mathrm{Q}$ of each signal together it will result in a signal without IQ mismatch in each branch as expressed in (5).

Two approaches are proposed for processing and tracking the orthogonal signal. The first approach is to feed independently the real and the imaginary parts of the complex signal into a separate PLL, as shown in Fig. 4. Where, the real part represents the first received signal and the imaginary part represents the second received signal. As required, there is no IQ mismatch in both signals. The second approach is to feed the signal into a single baseband CQPLL (after removing the carrier frequency as shown in Fig. 2) to track and decode the signal because both signals' information is available at the same time, but this needs to be solved in a reorthoganalised system between the two signals as shown in (6).

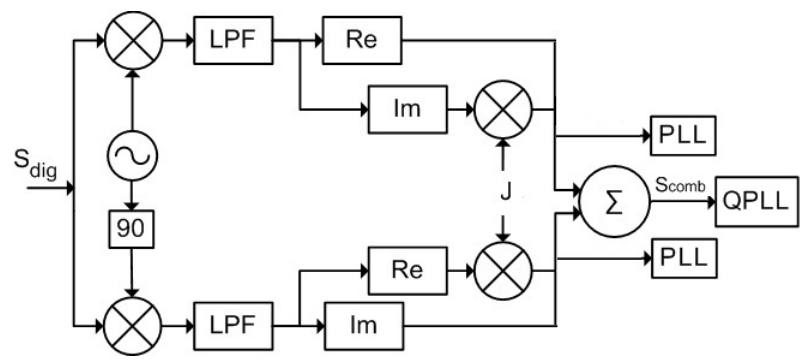

Figure 4. Removing the signals carrier in the digital domain

In addition, the OCBPSR can be used to cluster the spectrum of the received signals based on choosing the sampling frequency that folds each two signals to one specific band in the NZs.

Equations (1) and (2) represent two Binary Phase Shift Keying (BPSK) bandpass signals $\mathrm{S} 1$ and $\mathrm{S} 2$ that are received through fading channel.

$$
\begin{aligned}
& S_{1}=A_{1} \cos \left(2 \pi\left(f_{1}+f_{d 1}\right) t+\varphi_{1}\right)+n_{1} \\
& S_{2}=A_{2} \cos \left(2 \pi\left(f_{2}+f_{d 2}\right) t+\varphi_{2}\right)+n_{2}
\end{aligned}
$$

Where, $\left(A_{1}, f_{c 1}, f_{d 1}, n_{1}\right)$ and $\left(A_{2}, f_{c 2}, f_{d 2}, n_{2}\right)$ represent the amplitude, the carrier frequency, the Doppler frequency and the Gaussian noise of the first and second signals respectively. $\varphi_{1}$ and $\varphi_{2}$ represent the information message of the first and second signals respectively, and can be expressed as:

$$
\begin{aligned}
\varphi_{1} & =\pi\left(1-b_{1}\right), \text { where } b_{1}=0,1 \text { bits } \\
\varphi_{2} & =\pi\left(1-b_{2}\right), \text { where } b_{2}=0,1 \text { bits }
\end{aligned}
$$

As described earlier, by applying HT to (2), then summing with (1) will generate the analytic signal that will be folded to the FNZ as shown in (3). Let us assume the HT impairment in this implementation is $\gamma$ in phase and $\alpha$ in amplitude.

For simplifying the system derivation let us assume that the signals have the same relative power and amplitudes of the signals equal one.

$$
\begin{aligned}
\mathrm{S}_{\mathrm{dig}}=\mathrm{m}_{1} \cos \left(2 \pi n \mathrm{Tf}_{\text {fold }}+\beta_{1}\right) & \\
& +\mathrm{j} \alpha\left(\mathrm{m}_{2} \sin \left(2 \pi n \mathrm{Tf}_{\text {fold }}+\gamma \beta_{2}\right)\right)+\mathrm{N}
\end{aligned}
$$

Where, $\mathrm{S}_{\mathrm{dig}}$ is the orthogonal complex digital signal at the fold-frequency $\left(f_{\text {fold }}\right)$ and it carries two different information massages $\mathrm{m}_{1}= \pm 1$ and $\mathrm{m}_{2}= \pm 1$ and $\beta_{1}=2 \pi n \mathrm{nf}_{\mathrm{d} 1} \&$ $\beta_{2}=2 \pi n \mathrm{Tf}_{\mathrm{d} 2}$, where $\mathrm{N}$ represents the combined noise.

By rewriting (3) we can see the complex signal is orthogonalised at $\mathrm{f}_{\text {fold }}$ frequency:

$$
\begin{aligned}
& \mathrm{S}_{\mathrm{dig}} \\
& =\left[\mathrm{m}_{1} \cos (\beta 1)+\mathrm{j} \alpha \mathrm{m} 2 \sin \left(\gamma \beta_{2}\right)\right] \cos \left(2 \pi \mathrm{nTf}_{\text {fold }}\right) \\
& +[-\mathrm{m} 1 \sin (\beta 1)+\mathrm{j} \alpha \mathrm{m} 2 \cos (\gamma \beta 2)] \sin \left(2 \pi \mathrm{nf}_{\text {fold }}\right)
\end{aligned}
$$

After removing the carrier and recombining the real and imaginary parts of the signals as depicted in Fig. 2, the resulting signal is given by:

$$
S_{c o m b}=\left(m_{1} e^{j \beta_{1}}+j \alpha m_{2} e^{j \gamma \beta_{2}}\right)
$$

Further processing is requiring for tracking the $\mathrm{S}_{\text {comb }}$ signal in the second approach. That will require solving the Doppler frequency difference in the two signals. Equation (6) expresses the mathematical system that can solve the difference.

$$
\left[\begin{array}{l}
\mathrm{m} 1 \\
\mathrm{~m} 2
\end{array}\right]=\left[\begin{array}{ll}
1 & \alpha \sin (\beta) \\
0 & \alpha \cos (\beta)
\end{array}\right]\left[\begin{array}{l}
\mathrm{I} \\
\mathrm{Q}
\end{array}\right]
$$

Where, $\beta$ is the difference between $\beta_{1}$ and $\gamma \beta_{2}$. I and $Q$ represent the in-phase and quadrature-phase components of the CQPLL.

\section{OCBPSR FADING CHANNELS}

A typical transmitted signal over a wireless channel will suffer from various "fading" phenomena such as Doppler effects, multipath, path loss, shadowing, etc. For signal analysis, there are two models of fading channel representations. These are: 1) The Large-scale fading channel is characterized mostly by the degradation of the signal power due to shadowing by large objects such as buildings and hills as well as path loss of signal over a large distance between the transmitter and receiver. However, this type of fading has a slow fluctuation effect on the signal strength because fluctuations occur when the receiver moves over many wavelengths of the signal carrier. 2) The Small-scale fading channel refers to rapid fluctuations of the amplitude and phase of the received signals due to constructive and destructive interference among signals that arrive at the receiver at different times. There are two Small-scale fading types; A) "Fast-fading" has a high Doppler spread and a channel coherence time (commonly defined as the time in which the channel can be considered constant) less than the symbol 
period. i.e. channel variations are faster than baseband signal variations. B) "Slow-fading" has a low Doppler spread and the channel coherence time is greater than the symbol period. i.e. the channel variations are slower than the baseband signal variations.

For our simulation, two types of Small-scale Slow-fading channels are used. These are a frequency-flat fading channel and frequency-selective fading channel. Typically, in timedomain a channel characterises as a flat fading when a multipath delay spread (defined as the difference in propagation time between the longest and shortest path of the received signal) is less than the symbol period. Correspondingly, in frequency-domain, the bandwidth of the received signal is less than a coherence bandwidth of the channel, which is inversely related to the value of delay spread. A channel becomes frequency-selective when the delay spread is larger than the symbol duration, i.e. the bandwidth of the received signal is larger than a coherence bandwidth.

\section{EXPERIMENTAL SET-UP \& RESULT}

MATLAB is used for simulating the OCBPSR implementation. To represent the transmitted signals, two BPSK are modulated signals with $1 \mathrm{MHz}$ and $0.5 \mathrm{MHz}$ bandwidths representing the first and the second signal respectively. These signals are passed through a "root raised cosine filter" with a roll-off factor of 0.25 . Two different carrier frequencies of $900 \mathrm{MHz}$ and $850 \mathrm{MHz}$ are used for the first signal and the second signal respectively. A Rician fading (frequency-selective and flat fading) is then used to simulate transmission channel. The frequency-selective channel characteristic parameters are shown in table I.

TABLE I. PARAMERTR FOR FREQUENCY-SELECTIVE FADING CHANNE

\begin{tabular}{|l|l|l|l|}
\hline Key Channel Parameters & Symbol & $\begin{array}{l}\text { Values of } \\
\text { 1st Signal }\end{array}$ & $\begin{array}{l}\text { Values of } \\
\text { 2nd } \\
\text { Signal }\end{array}$ \\
\hline Carrier frequency & $\mathrm{f}_{\mathrm{c}}$ & $900 \mathrm{MHz}$ & $850 \mathrm{MHz}$ \\
\hline Communcation bandwidth & $\mathrm{W}$ & $1 \mathrm{MHZ}$ & $0.5 \mathrm{MHz}$ \\
\hline Velocity of mobile & $\mathrm{V}$ & $70 \mathrm{Km} / \mathrm{h}$ & $70 \mathrm{Km} / \mathrm{h}$ \\
\hline Doppler shift for a path & $\mathrm{D}$ & $60 \mathrm{~Hz}$ & $55 \mathrm{~Hz}$ \\
\hline Coherence time & $\mathrm{T}_{\mathrm{c}}=1 /(2 \mathrm{D})$ & $8.3 \mathrm{~ms}$ & $9.1 \mathrm{~ms}$ \\
\hline Dealy spread & $\mathrm{T}_{\mathrm{d}}$ & $2.2 \mu \mathrm{s}$ & $4 \mu \mathrm{s}$ \\
\hline Coherence bandwith & $\mathrm{W}_{\mathrm{c}}$ & $500 \mathrm{KHz}$ & $250 \mathrm{KHz}$ \\
\hline
\end{tabular}

These two simulated signals once captured by their respective antennas, are passed through LNAs and two narrow BPF's centred on carrier frequencies of $900 \mathrm{MHz}$ and 850 $\mathrm{MHz}$ to eliminate all frequencies outside the signals bandwidth. The resulting in-band signals are then fed to the OIF. Note that the phase of the second signal is shifted 900 (Add random phase and gain to the signal to cause imbalance between in-phase and quadrature) and digitised in parallel with the first signal by two of the ADCs that run in the same sampling frequency at $7 \mathrm{MHz}$. This sampling frequency is chosen so that the ADCs fold the two signals directly to the analytic signal at $3 \mathrm{MHz}$ fold-frequency. The output complex orthogonal digital signal can then be tracked and demodulated through two digital approaches, as we explained earlier.

Regarding the second approach, RLS and LSM adaptive algorithms (Equalizer) are chosen individually to solve the reorthoganalised system (6). These algorithms are integrated inside the loop of the CQPLL to re-orthogonalise its branches. Note that the PLL, CQPLL and the equalizer algorithms are implemented in MATLAB too.

BER and EVM are used for evaluating the simulation result of the OCBPSR performance. BER is one of the measurement tools used to evaluate end-to-end communication systems based on comparing the transmitted and received bits. On the other hand the EVM is defined as the difference between the estimated complex voltage of the demodulated symbol and the value of the actual received symbol.

The simulation results show that the BER and the EVM of the flat and frequency-selective channels are almost identical in the first approach. Therefore, the simulation results of the frequency-selective channel are discussed. Fig. 5 illustrates that the BER curves of the demodulated signals are approximately similar to the theoretical curve. This proves that tracking and decoding the signals in the separate PLLs are done properly.

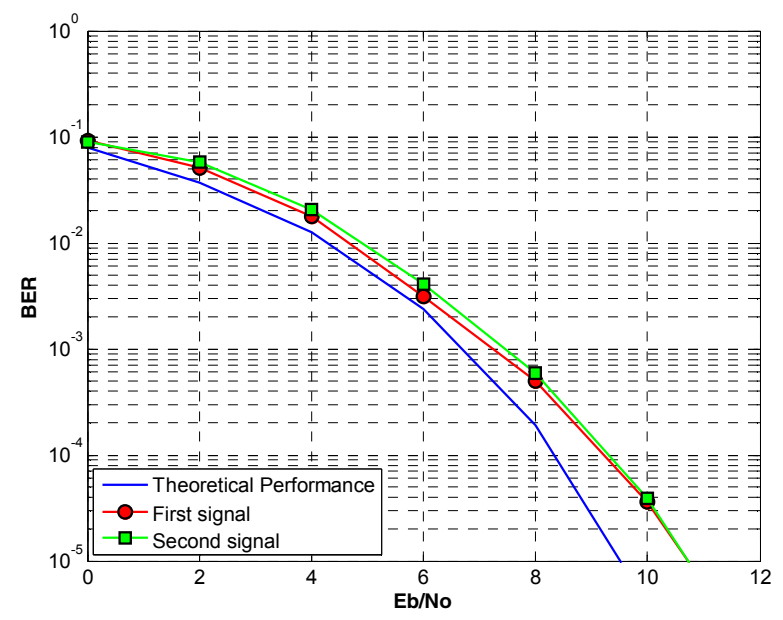

Figure 5. BER vs Eb/No in frequency-selective channel based in two PLLs

Table II shows further indication of recovering the I and Q of the signals so that they are perfectly isolated between the signals in the digital domain without suffering from overlapping and the IQ mismatch.

TABLE II. EVM VALUES OF DEMODULATED SIgNALS

\begin{tabular}{|l|l|l|}
\hline & EVM $_{\text {RMS }}$ & EVM max peak at symbol \\
\hline Signal 1 & $2.02 \%$ & $9.36 \%$ \\
\hline Signal 2 & $2.11 \%$ & $9.62 \%$ \\
\hline
\end{tabular}

In the second approach we will track and demodulate the complex orthogonal signals in single CQPLL. Fig. 6 displays the BER curves of demodulated signals in the frequencyselective channel. The curves of LMS and RLS have an acceptable increase compared with theoretical curve. Besides, these curves are approximately identical to each other, while the curve of demodulated signal without utilizing the equalizer 
algorithms is far from the acceptable values. These results are expected as the received signals have different Doppler frequency so the CQPLL cannot track them correctly, without employing the algorithms. Note that the CQPLL is tracking the Doppler frequency change of the first signal and solving the Doppler frequency of the second signal.

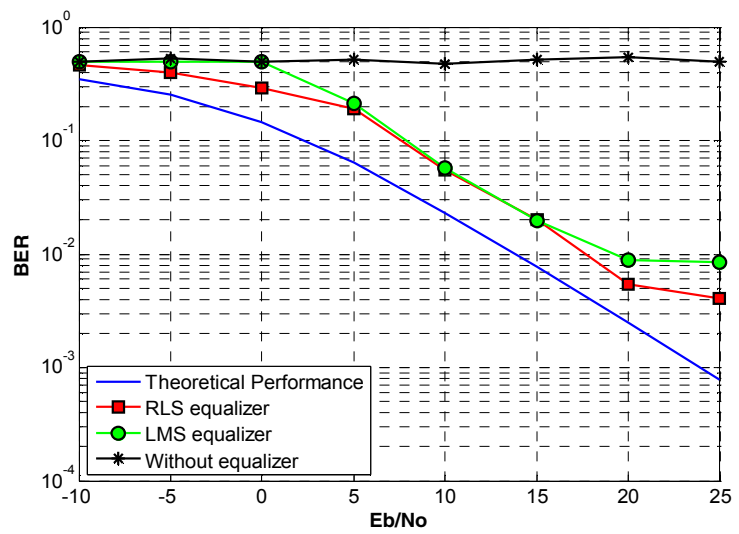

Figure 6. BER vs Eb/No in frequency-selective channel, single CQPLL

Now we analyse further the demodulated signals in the frequency-selective channel in terms of EVM. The simulated measurement value of EVM in Fig. 7 shows both the algorithms have the same performance. The EVM is increasing perfectly with the increasing SNR. This proves that the phase and the amplitude of the both signals are re-orthogonalising well based on the chosen algorithms, which also demonstrates that the front-end is perfectly orthogonalising the signals in the fold-frequency.

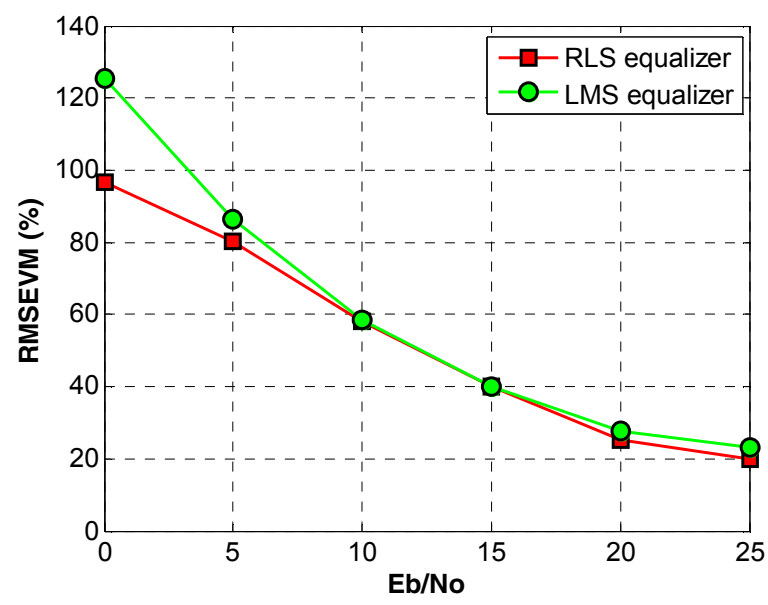

Figure 7. EVM vs Eb/No in frequency-selective channel, single CQPLL

Fig. 8 shows the scattering plot of the signals. It is clear that the analytic demodulated signal without using the algorithms (with Doppler shift), has wrong value of phase and amplitude with respect to the actual reference signal value. Besides, the figure shows the two algorithms perfectly recover the correct phase and amplitude of the demodulated signal.

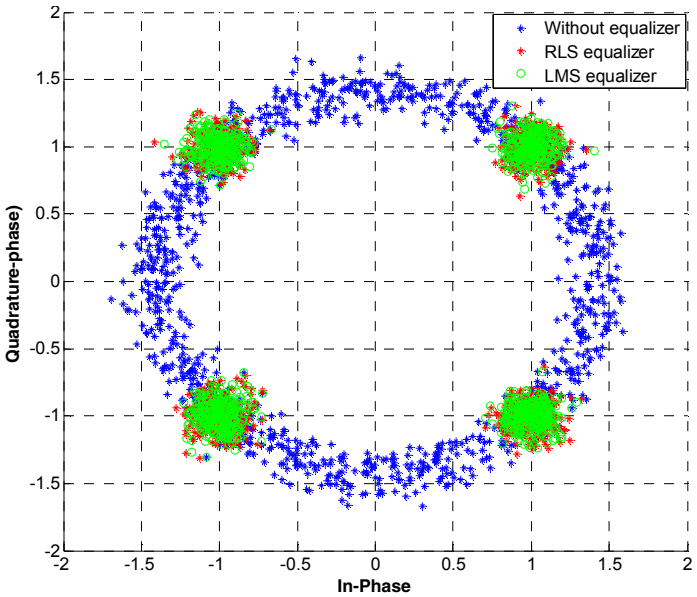

Figure 8. Scattering plot of signals demodulated in single CQPLL

The same evaluation has been done to the flat fading channel. Fig. 9 demonstrates a small raise in the value of the BER of the OCBPSR compared with the theoretical performance. It also shows that the performances of the two algorithms are almost identical in the flat channel and have better performance than the frequency-selective fading channel.

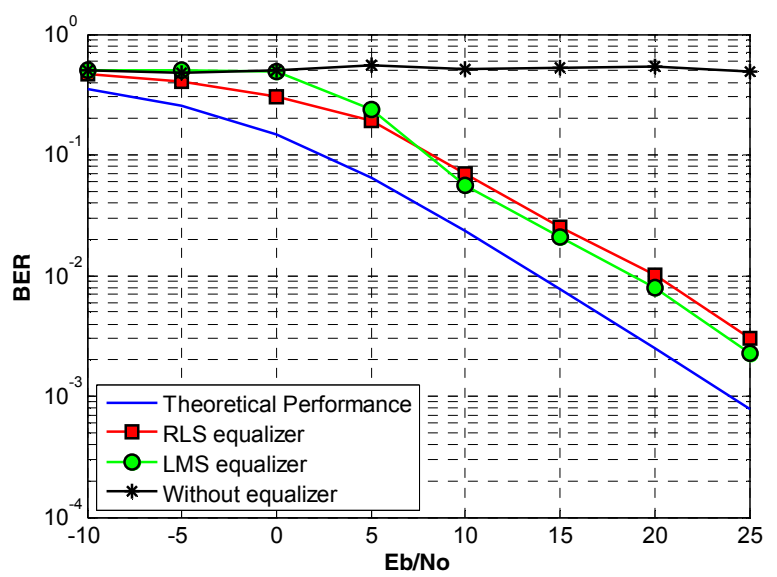

Figure 9. BER vs Eb/No in flat channel, single CQPLL

Fig. 10 shows EVM of the demodulated symbol in both signals in the flat channel and it is approximately similar to the value of the actual received symbol with the increasing SNR. Generally, the BER and the EVM values in the flat channel are better estimated compared with the frequency-selective channel. That is expected because the frequency-selective channel is more challenging than the flat channel because the bandwidth of the received signals is larger than a coherence bandwidth. 


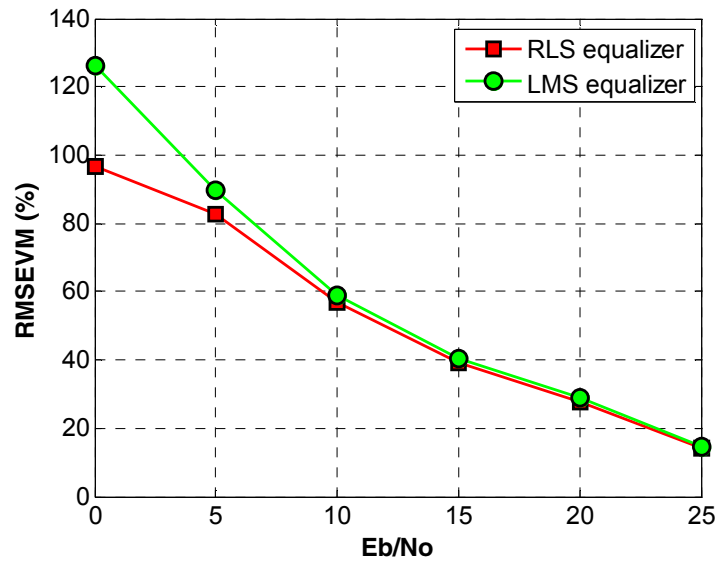

Figure 10. EVM vs Eb/No in flat channel, single CQPLL

\section{CONCLUSION}

This paper presents a new multi-signal receiver based on second-order BPS that has the ability to capture and track two signals at the same time. The implementation of this receiver ensures that there is no IQ mismatch in the signals. In addition it will reduce the sampling frequency to a rate proportional to the maximum bandwidth information of the input signals, and therefore requiring less processing time in the digital domain of the receiver.

Two approaches have been proposed to track and decode these two signals. The first one utilises two baseband PLLs to track a single-side band signals, while the second approach uses a single baseband CQPLL for tracking and decoding the two signals simultaneously.

The simulation results shows that the OCBPSR has a good performance all throughout handling and tracking the signals under different scenarios of harsh signal environment.

\section{ACKNOWLEDGMENT}

The researchers would like to thank Iraqi ministry of higher education for the PhD scholarship given to the first research.

\section{REFERENCES}

[1] C. Gernot, K. O'Keefe and G. Lachapelle, "Combined L1/L2C tracking scheme for weak signal environments," 2008.

[2] M. Al-Aboodi, A. Albu-Rghaif and I. A. Lami, "GPS, Galileo and Glonass L1 signal detection algorithms based on bandpass sampling techniques," in IEEE, 2012.

[3] J. M. III and G. Q. Maguire, "Cognitive radio: making software radios more personal," Personal Communications, IEEE, vol. 6, no. 4, pp. 13$18,1999$.

[4] T. Fujii, Y. Kamiya and Y. Suzuki, "Multi-band ad-hoc cognitive radio for reducing inter system interference," in IEEE, 2006.

[5] R. G. Vaughan, N. L. Scott and D. R. White, "The theory of bandpass sampling," Signal Processing, IEEE Transactions on, vol. 39, no. 9, pp. 1973-1984, 1991.

[6] D. M. Akos, M. Stockmaster, J. B. Tsui and J. Caschera, "Direct bandpass sampling of multiple distinct RF signals," Communications, IEEE Transactions on, vol. 47, no. 7, pp. 983-988, 1999.

[7] M. Valkama, Advanced I/Q signal processing for wideband receivers: Models and algorithms, 2001.

[8] D. Sanderson, R. Svitek and S. Raman, "A 5-6-GHz polyphase filter with tunable I/Q phase balance," Microwave and Wireless Components Letters, IEEE, vol. 14, no. 7, pp. 364-366, 2004.

[9] G.-T. Gil, I.-H. Sohn, J.-K. Park and Y. H. Lee, "Joint ML estimation of carrier frequency, channel, I/Q mismatch, and DC offset in communication receivers," Vehicular Technology, IEEE Transactions on, vol. 54, no. 1, pp. 338-349, 2005.

[10] I.-H. Sohn, E.-R. Jeong and Y. H. Lee, "Data-aided approach to I/Q mismatch and DC offset compensation in communication receivers," Communications Letters, IEEE, vol. 6, no. 12, pp. 547-549, 2002.

[11] M. Valkama, M. Renfors and V. Koivunen, "Blind source separation based I/Q imbalance compensation," 2000.

[12] M. Valkama, M. Renfors and V. Koivunen, "Compensation of frequency-selective I/Q imbalances in wideband receivers: models and algorithms," 2001.

[13] W. R. Dieter, S. Datta and W. K. Kai, "Power reduction by varying sampling rate," in $A C M, 2005$.

[14] D. Akos, M. Stockmaster, J. Tsui and J. Caschera, "Direct bandpass sampling of multiple distinct RF signals," Communications, IEEE Transactions on, vol. 47, no. 7, pp. 983-988, jul 1999.

[15] I. Lami and M. Ai-Aboodi, "OBPSR: A multi-signal receiver based on the orthogonal and bandpass sampling techniques," 2013. 